\title{
Flavokawain derivative FLS induced G2/M arrest and apoptosis on breast cancer MCF-7 cell line
}

This article was published in the following Dove Press journal:

Drug Design, Development and Therapy

10 June 2016

Number of times this article has been viewed

\author{
Norlaily Mohd Ali ${ }^{1}$ \\ M Nadeem Akhtar ${ }^{2}$ \\ Huynh $\mathrm{Ky}^{3}$ \\ Kian Lam Lim' \\ Nadiah $\mathrm{Abu}^{4}$ \\ Seema Zareen ${ }^{2}$ \\ Wan Yong $\mathrm{Ho}^{5}$ \\ Han Kiat Alan-Ong' \\ Sheau Wei Tan ${ }^{6}$ \\ Noorjahan Banu Alitheen ${ }^{4}$ \\ Jamil bin Ismail ${ }^{2}$ \\ Swee Keong Yeap 6 \\ Tunku Kamarul ${ }^{7}$ \\ 'Faculty of Medicine and Health \\ Sciences, Universiti Tunku Abdul \\ Rahman, Selangor, ${ }^{2}$ Department of \\ Industrial Biotechnology, Faculty of \\ Industrial Sciences \& Technology, \\ Universiti Malaysia Pahang, Pahang, \\ Malaysia; ${ }^{3}$ Department of Agriculture \\ Genetics and Breeding, College of \\ Agriculture and Applied Biology, \\ Cantho University, CanTho City, \\ Vietnam; ${ }^{4}$ Department of Cell \\ and Molecular Biology, Faculty of \\ Biotechnology and Biomolecular \\ Sciences, Universiti Putra Malaysia, \\ ${ }^{5}$ School of Biomedical Sciences, The \\ University of Nottingham Malaysia \\ Campus, 'Institute of Bioscience, \\ Universiti Putra Malaysia, Selangor, \\ ${ }^{7}$ Tissue Engineering Group, National \\ Orthopaedic Centre of Excellence for \\ Research and Learning, Department \\ of Orthopaedic Surgery, Faculty of \\ Medicine, University Malaya, Kuala \\ Lumpur, Malaysia
}

Correspondence: Swee Keong Yeap Institute of Bioscience, Lebuh Silicon, Universiti Putra Malaysia, 43400 Serdang, Selangor, Malaysia

Tel +60389472153

Fax +60 389472153

Email skyeap2005@gmail.com
Abstract: Known as naturally occurring biologically active compounds, flavokawain A and $\mathrm{B}$ are the leading chalcones that possess anticancer properties. Another flavokawain derivative, (E)-1-(2'-Hydroxy-4',6'-dimethoxyphenyl)-3-(4-methylthio)phenyl)prop-2-ene-1-one (FLS) was characterized with ${ }^{1} \mathrm{H}$-nuclear magnetic resonance, electron-impact mas spectrometry, infrared spectroscopy, and ultraviolet ( ${ }^{1} \mathrm{H}$ NMR, EI-MS, IR, and UV) spectroscopic techniques. FLS cytotoxic efficacy against human cancer cells (MCF-7, MDA-MB-231, and MCF-10A) resulted in the reduction of $\mathrm{IC}_{50}$ values in a time- and dose-dependent mode with high specificity on MCF-7 ( IC $_{50}$ of $36 \mu \mathrm{M}$ at 48 hours) against normal breast cell MCF-10A (no $\mathrm{IC}_{50}$ detected up to $180 \mu \mathrm{M}$ at 72 hours). Light, scanning electron, and fluorescent microscopic analysis of MCF-7 cells treated with $36 \mu \mathrm{M}$ of FLS displayed cell shrinkage, apoptotic body, and DNA fragmentation. Additionally, induction of G2/M cell arrest within 24 hours and apoptosis at subsequent time points was discovered via flow cytometry analysis. The roles of $P L K-1$, Wee-1, and phosphorylation of CDC-2 in G2/M arrest and proapoptotic factors (Bax, caspase 9, and p53) in promotion of apoptosis of FLS against MCF-7 cells were discovered using fluorometric, quantitative real-time polymerase chain reaction, and Western blot analysis. Interestingly, the presence of $\mathrm{SCH}_{3}$ (thiomethyl group) on ring B structure contributed to the selective cytotoxicity against MCF-7 cells compared to other chalcones, flavokawain A and B. Overall, our data suggest potential therapeutic value for flavokawain derivative FLS to be further developed as a new anticancer drug.

Keywords: (E)-1-(2'-Hydroxy-4',6'-dimethoxyphenyl)-3-(4-methylthio)phenyl)prop-2-ene-1one (FLS), MCF-7, G2/M arrest, apoptosis, cell cycle, $P L K-1$, p53, caspase

\section{Introduction}

Breast cancer is the leading cause of cancer death in females worldwide, accounting for $\sim 25 \%$ ( 1.68 million cases $)$ of the total new cancer cases and $\sim 14 \%$ (521,900 deaths) of the total cancer deaths in 2012.1,2 Among the various types of cancer, breast cancer is the most frequently diagnosed and the most common cancer that affects Malaysian women from all ethnicities. ${ }^{3}$ According to recent reports, an estimated 232,670 new cases of breast cancer have been expected and diagnosed among women and about 2,360 new cases in men in the US during 2014. ${ }^{4,5}$ Although numerous efforts have been made to decrease the mortality rate of breast cancer, ${ }^{6}$ to-date, no ultimate chemotherapeutic drug is available to overcome this disease. Thus, effort in searching alternative or better candidates of antibreast cancer compound from natural phytochemical or synthetic chemistry is still ongoing. ${ }^{7}$

A number of studies have reported the broad spectrum bioactivities of chalcones including antioxidant, cytotoxic, anti-inflammation, antihistaminic, and antimicrobial effects. ${ }^{8}$ Flavokawains (either A, B, or C) are among the well-studied chalcones that 
were extracted from Piper methysticum. Flavokawain A (FLA) constituted as the highest amount of flavokawain found in kava extract, while flavokawain B (FLB) is the most well studied flavokawain. Both flavokawain possess various level of cytotoxicity against several cancer cell line including colon, bladder, and breast. ${ }^{9-12}$ An ideal cytotoxic compound should possess good selectivity against the targeted cancer cell while inducing limited or no toxicity against normal cell. ${ }^{9}$ Comparing the cytotoxic effect of FLA and FLB, these two compounds were recorded with different degrees of in vitro cytotoxicity against estrogen-dependent MCF-7 (FLA: $\sim 25 \mu \mathrm{M}$; FLB: $\sim 33 \mu \mathrm{M}$ ) and estrogen-independent MDA-MB-231 (FLA: 17 $\mu \mathrm{M}$; FLB: $\sim 12 \mu \mathrm{M}$ ) breast cancer cell lines. However, both flavokawains also exhibited cytotoxicity on normal MCF-10A (FLA: 95 $\mu \mathrm{M}$; FLB: $~ 45 \mu \mathrm{M}$ ) breast cell line. ${ }^{9,10}$ Synthesis of bioactive compounds allows the possibility of modifying the lead compound for better efficacy and desired cytotoxic action. For example, Badisa et $\mathrm{al}^{7}$ have shown that synthetic piperidinyl-diethylstilbestrol and pyrrolidinyl-diethylstilbestrol possessed better selectivity against breast cancer MCF-7 cell than normal breast MCF$10 \mathrm{~A}$ cell. In this investigation, a flavokawain derivative, (E)-1-(2-hydroxy-4,6-dimethoxyphenyl)-3-(4-(methylthio) phenyl)prop-en-1-one (FLS) was synthesized via a ClaisenSchmidt condensation reaction. Thus, the aim of this study was to compare the selectivity of flavokawain derivative FLS on breast cancer and normal cell lines. In addition, the regulation of cell cycle and apoptotic inducing effect of FLS against MCF-7 were also observed.

\section{Materials and methods}

\section{Synthesis of FLS}

FLS was synthesized via a Claisen-Schmidt condensation reaction. Acetophenone, 4,6-dimethoxy-2'-hydroxyacetophenone $3.0 \mathrm{~g}(9.0 \mathrm{mmol})$ and 4-(methylthio)benzaldehyde $(1.4 \mathrm{~mL}$, $10 \mathrm{mmol})$ were dissolved in methanol. A 40\% water solution of potassium hydroxide $5.0 \mathrm{~g}$ and $50 \mathrm{~mL}$ methanol was mixed in a $500 \mathrm{~mL}$ round bottle flask and left for 15 minutes at room temperature. The crude product was transferred into separating funnel $(500 \mathrm{~mL})$ and then added with $200 \mathrm{~mL}$ distilled water. The mixture was then acidified with the addition of 2-3 mL hydrochloride. The product was extracted with $100 \mathrm{~mL}$ ethyl acetate. The organic layer was then successively washed with brine and dried over anhydrous sodium sulfate. The crude product was finally purified by flash column chromatography with ethyl acetate and hexane (5:5) as eluants and recrystallized in ethyl acetate and drops of $\mathrm{MeOH}$.

\section{MTT cell viability assay}

MCF-7, MDA-MB-231, and MCF-10A cells (American Type Culture Collection [ATCC], Manassas, VA, USA) were seeded overnight in 96-well plates at a concentration of $0.8 \times 10^{5}$ cells $/ \mathrm{mL}$ at $37^{\circ} \mathrm{C} \mathrm{CO}_{2}$ incubator in Roswell Park Memorial Institute medium-1640, Dulbecco's Modified Eagle's Medium (DMEM), and DMEM-F12 supplemented with hydrocortisone $(0.5 \mu \mathrm{g} / \mathrm{mL})$, insulin $(10 \mu \mathrm{g} / \mathrm{mL})$, human epidermal growth factor $(20 \mathrm{ng} / \mathrm{mL})$ (Sigma-Aldrich Co., St Louis, MO, USA) medium, respectively. Then, respective cells were treated with FLS (ranging between 180 and $3 \mu \mathrm{M}$ ) for 24,48 , and 72 hours. MTT solution $(5 \mathrm{mg} / \mathrm{mL})$ was added to all wells 3 hours before end of incubation time. Absorbance was read at wavelength of $570 \mathrm{~nm}$ (BioTek Instruments, Winooski, VT, USA). ${ }^{13}$ Percentage viability of the tested cell line was calculated using the following formula:

$$
\begin{aligned}
& \text { Cell } \\
& \text { viability (\%) }
\end{aligned}=\frac{\text { Absorbance sample }}{\text { Absorbance untreated control }} \times 100 \%
$$

$\mathrm{IC}_{50}$ value indicating concentration that inhibited $50 \%$ of the tested cell lines was obtained from the graph plotted with percentage of cell viability against concentration of FLS.

\section{MCF-7 cell treatment}

Based on the MTT assay (Table 1), reduction in $\mathrm{IC}_{50}$ values of FLS was observed after treatment at 24-72 hours on MCF-7

Table I Comparison values of $\mathrm{IC}_{50}$ of FLS, FLA, and FLB in MCF-7, MDA-MB-23I and MCF-IOA cells

\begin{tabular}{|c|c|c|c|c|c|}
\hline \multirow[t]{2}{*}{ Cell lines } & \multicolumn{3}{|l|}{ FLS $(\mu \mathrm{M})$} & \multirow{2}{*}{$\frac{\text { FLA }(\mu M)^{9}}{72 \text { hours }}$} & \multirow{2}{*}{$\frac{\text { FLB }(\mu M)(\text { Abu et al })^{10}}{72 \text { hours }}$} \\
\hline & 24 hours & 48 hours & 72 hours & & \\
\hline MCF-7 & $40.90 \pm 4.50$ & $36.36 \pm 3.10$ & $33.30 \pm 2.50$ & $25.13 \pm 3.00$ & $33.76 \pm 1.35$ \\
\hline MDA-MB-23I & $63.64 \pm 3.80$ & $51.52 \pm 4.20$ & $48.48 \pm 3.40$ & $17.49 \pm 1.50$ & $|2.3| \pm 1.22$ \\
\hline MCF-IOA & $>180$ & $>180$ & $>180$ & $>100$ & 45 \\
\hline Selective index of MCF-I0A/MCF-7 & $>4.40$ & $>4.95$ & $>5.41$ & 3.98 & 1.33 \\
\hline Selective index of MCF-I0A/MDA & $>2.83$ & $>3.49$ & $>3.71$ & 5.72 & 3.66 \\
\hline
\end{tabular}

Notes: Adapted from Abu N, Akhtar MN, Yeap SK, et al. Flavokawain A induces apoptosis in MCF-7 and MDA-MB23I and inhibits the metastatic process in vitro. PLoS One. 2014;9:e105244. ${ }^{9}$ Adapted from Abu N, Akhtar M, Yeap S, et al. Flavokawain B induced cytotoxicity in two breast cancer cell lines, MCF-7 and MDA-MB23 I and inhibited the metastatic potential of MDA-MB23I via the regulation of several tyrosine kinases in vitro. BMC Complem Altern M. 2016; I6:86. ${ }^{\circ}$

Abbreviations: FLS, (E)-I-(2'-Hydroxy-4',6'-dimethoxyphenyl)-3-(4-methylthio)phenyl)prop-2-ene-I-one; FLA, flavokawain A; FLB, flavokawain B. 
verifying that FLS effect is time- and dose-dependent. To standardize the treatment, one concentration of FLS $(36 \mu \mathrm{M})$ was used for the remaining assays. MCF-7 cell was seeded at $0.8 \times 10^{5}$ cells $/ \mathrm{mL}$ overnight in 6 -well plate. After reaching $80 \%-90 \%$ confluence, the cell was treated with $36 \mu \mathrm{M}\left(\mathrm{IC}_{50}\right.$ at 48 hours) of FLS for 24, 48, and 72 hours. Untreated control was prepared simultaneously. After 24,48 , or 72 hours of incubation, cell was harvested using TrypLE (Thermo Fisher Scientific, Waltham, MA, USA), washed with phosphate-buffered saline and subjected to the subsequent assays.

\section{Light and scanning electron microscopic examination}

Cell was subjected to microscopic examination using inverted light microscope (Nikon Corporation, Tokyo, Japan) and scanning electron microscope (SEM; JEOL, Tokyo, Japan) after 72 hours of treatment. Prior to SEM observation, cell was seeded on poly-L-lysine coated round coverslips in a 6-well plate. After 72 hours, untreated control and FLS treated MCF-7 cell were fixed in 4\% glutaraldehyde, washed with $0.1 \mathrm{M}$ sodium cocodylate buffer, fixed with $1 \%$ osmium tetroxide and underwent series of dehydration in acetone and dried using $\mathrm{CO}_{2}$. Then, the samples were mounted onto the stubbing, coated with gold in sputter and viewed under JEOL SEM under the $15 \mathrm{kV}$ setting.

\section{Acridine orange/propidium iodide dual staining}

Harvested cell was stained with $10 \mu \mathrm{g} / \mathrm{mL}$ of acridine orange and propidium iodide (PI) each. The morphology of cell was observed under fluorescent microscope (Nikon) with combination of excitation filter (450-490 nm) and long pass filter $(520 \mathrm{~nm})$. Random scoring of 200 cells for both untreated and FLS-treated MCF-7 was taken to determine the percentage of viable (green intact cell), apoptotic (green cell with condensed or fragmented nucleus), and necrotic (red cell) cells.

\section{Cell cycle analysis}

Cell cycle progression was evaluated using BD Cycletest Plus kit and subjected to BD FACS Calibur flow cytometer (BD, Franklin Lakes, NJ, USA) analysis. In brief, harvested cell was incubated with $250 \mu \mathrm{L}$ of trypsin buffer for 10 minutes followed by $200 \mu \mathrm{L}$ of trypsin inhibitor with RNase buffer. The samples were finally stained with $200 \mu \mathrm{L}$ of PI solution and analyzed.

\section{Annexin V/PI apoptosis study}

Apoptosis of cell was evaluated using BD Annexin V-fluorescein isothiocyanate (FITC)/PI apoptosis detection kit. In brief, harvested cell was washed and added with $100 \mu \mathrm{L}$ of $1 \times$ binding buffer. The cell was then stained with $5 \mu \mathrm{L}$ of Annexin V-FITC and $5 \mu \mathrm{L}$ of PI solution, incubated for 15 minutes and diluted with $400 \mu \mathrm{L}$ of $1 \times$ binding buffer. All samples were analyzed with BD FACS Calibur flow cytometer (Becton Dickinson).

\section{Quantitative reverse transcription real- time PCR assay}

RNA was extracted from cell using RNeasy mini plus kit (Qiagen NV, Venlo, the Netherlands). The purity and concentration of the isolated RNA were quantified prior to DNA conversion using iScript cDNA synthesis kit (Bio-Rad Laboratories Inc., Hercules, CA, USA). Expression of pololike kinase 1 (PLK1), WEE1 G2 checkpoint kinase (WEE-1), c-Jun proto-oncogene (c-Jun), Glucose transporter (GLUT) were quantified by quantitative real-time PCR (qRT-PCR) using SYBR select master mix (Thermo Fisher Scientific) on iQ-5 real-time polymerase chain reaction (PCR) machine (Bio-Rad) with normalization to three housekeeping genes, $\beta$-actin, 18srRNA, and GAPDH. All primers are listed in Table 2. PCR condition for this experiment was 1 cycle of $50^{\circ} \mathrm{C} / 2$ minutes for Uracil-DNA glycosylase (UDG) activation; 1 cycle of $95^{\circ} \mathrm{C} / 2$ minutes for DNA polymerase

Table 2 The accession number and sequence of the primers used in the quantitative real-time PCR assay

\begin{tabular}{|c|c|c|}
\hline Accession number & Gene & Sequence \\
\hline \multirow[t]{2}{*}{ NM_00II0I.3 } & ACTB & F: 5'-AGAGCTACGAGCTGCCTGAC-3' \\
\hline & & R: 5'-AGCACTGTGTTGGCGTACAG-3' \\
\hline \multirow[t]{2}{*}{ NM_002046.4 } & GAPDH & F: 5-GGATTTGGTCGTATTGGGC-3 \\
\hline & & R: 5-TGGAAGATGGTGATGGGATT-3 \\
\hline \multirow[t]{2}{*}{ HQ387008.I } & I8S rRNA & F: 5-GTAACCCGTTGAACCCCATT-3 \\
\hline & & R: 5-CCATCCAATCGGTAGTAGCG-3 \\
\hline \multirow[t]{2}{*}{ NM_005030.3 } & PLKI & F: 5-CCTGCACCGAAACCGAGTTAT-3 \\
\hline & & R:5-CCGTCATATTCGACTTTGGTTGC-3 \\
\hline \multirow[t]{2}{*}{ NM_00I I43976.I } & WEE-I & F: 5-GGGAATTTGATGTGCGACAG-3 \\
\hline & & R:5-CTTCAAGCTCATAATCACTGGCT-3 \\
\hline \multirow[t]{2}{*}{ NM_0065I6.2 } & GLUTI & F: 5-ACAACACTGGAGTCATCAATGC-3 \\
\hline & & R: 5-CCACAGAGAAGGAGCCAATCA-3 \\
\hline
\end{tabular}


activation; 40 cycles of $95^{\circ} \mathrm{C} 2$ seconds for denature, $60^{\circ} \mathrm{C}$ for 30 seconds for anneal and extend. Normalization of reference genes was carried out using geNorm algorithm.

\section{Western blot analysis}

Harvested cell was lysed with radioimmunoprecipitation assay (RIPA) buffer supplemented with phosphatase inhibitor cocktail (Hoffman-La Roche Ltd., Basel, Switzerland) prior to protein quantification using Bradford assay (Sigma-Aldrich Co.). Next, $100 \mu \mathrm{g}$ of cell lysate was electrophoresed in $10 \%$ sodium dodecyl sulfate polyacrylamide gel electrophoresis (SDS-PAGE) (Bio-Rad), transferred to nitrocellulose membrane using Pierce Fast Semi-Dry Blotter (Pierce, Thermo Fisher Scientific, Waltham, MA, USA), blocked with $0.5 \%$ skimmed milk and incubated with primary antibodies (antiCDC2, anti-p-CDC2, anti-p53, anti-Bax, anti-Bcl-2, and anti$\beta$-actin at dilution 1:1,000) (Abcam, San Francisco, CA, USA) for 1 hour. Following that, membrane was washed, incubated with goat anti-rabbit immunoglobulin G (IgG) and haematoxylin and eosin stain (H\&E) conjugated to alkaline phosphatase (Abcam) in 1:5,000 dilution and developed under chemiluminescence condition (SuperSignal West Pico, Pierce) using ChemiDoc XRS (Bio-Rad). The bands, intensity was analyzed using Quantity One 1D analysis software (Bio-Rad).

\section{Caspase 9 and cytochrome c detection study}

Extracted protein from previous Western blot analysis was also subjected to detection of caspase 9 and cytochrome c using CaspGLOW Red Active Caspase-9 staining kit (BioVision, Milpitas, CA, USA) and human cytochrome c platinum enzyme-linked immunosorbent assay (ELISA) (eBioscience
Affymetrix, Santa Clara, CA, USA). The fluorescence intensity in active caspase 9 was measured at $\mathrm{Ex} / \mathrm{Em}=540 / 570 \mathrm{~nm}$ using microplatefluorometer (Thermo Scientific). On the other hand, absorbance for human cytochrome c was measured using ELISA plate reader at wavelength of $450 \mathrm{~nm}$ (BioTek Instruments). Fold change of caspase 9 and cytochrome c were calculated by dividing fluorescence intensity or absorbance of FLS treated MCF-7 with untreated control MCF-7.

\section{Statistical analysis}

The data are presented as average of mean \pm SE (standard error of the mean), one-way analysis of variance was used to assess differences between time point groups. Results were considered statistically significant when $P<0.05$.

The Ethical Committee for Research in Universiti Putra Malaysia, decided that ethical approval was unnecessary for using commercial human cell lines.

\section{Results \\ Synthesis of FLS}

FLS was the yellow flat crystals with $78.6 \%$ yield through the synthesis. IR $\left(\mathrm{CHCl}_{3}\right): 1,642-1,644(\mathrm{C}=\mathrm{O}), 1,550-1,568$ $(\mathrm{C}=\mathrm{C}-\mathrm{C}=\mathrm{O}), 1,458-14,728(\mathrm{C}=\mathrm{C}, \mathrm{Ar})$, and 1,200-1,100 (C-O) $\mathrm{cm}^{-1} .{ }^{1} \mathrm{HNMR}\left(\mathrm{CDCl}_{3}, 500 \mathrm{MHz}\right): \delta 14.30$ (chelated

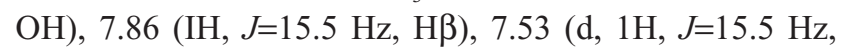
$\mathrm{H \alpha}$ ), 7.50 (br, d, 2H, H-2,6), 7.26 (m, 3H, H-3,4,5), 5.95 (d, J=2.5 Hz, 1H, H-3'), 5.90 (d, 1H, J=2.5 Hz, H-5'), 3.90 (s, 3H, OMe, C-6'), 3.82 (s, 3H, OMe, C-4'), 2.54 (s, 3H, $\mathrm{S}-\mathrm{CH}_{3}$ ). Electron-Impact Mas Spectrometry has indicated that synthesised FLS has molecular weight of 330.32 with molecular formula $\mathrm{C}_{18} \mathrm{H}_{18} \mathrm{O}_{4} \mathrm{~S}$. Molecular structures of FLS, FLA, and FLB are shown in Figure 1.<smiles>COc1ccc(/C=C/C(=O)c2c(O)cc(OC)cc2OC)cc1</smiles>

Flavokawain A

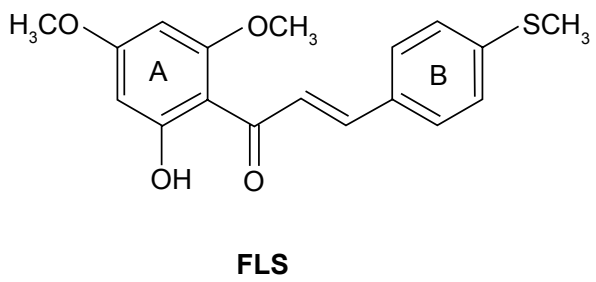<smiles>COc1cc(O)c(C(=O)/C=C/c2ccccc2)c(OC)c1</smiles>

\section{Flavokawain B}

Figure I The molecular structures of FLS, flavokawain A and flavokawain B.

Abbreviation: FLS: (E)-I-(2'-Hydroxy-4',6'-dimethoxyphenyl)-3-(4-methylthio)phenyl)prop-2-ene-I-one. 


\section{FLS selectively reduced the viability of MCF-7 cell}

FLS provoked a time-dependent cytotoxicity against both MCF-7 and MDA-MB-231 cells with 50\% reduction in a reducing concentration from 24 to 72 hours. The $\mathrm{IC}_{50}$ value of FLS on MDA-MB-231 was 1.5-fold higher than on MCF-7 indicating that FLS is more sensitive to MCF-7 $\left(\mathrm{IC}_{50} \sim 33 \mu \mathrm{M}\right)$ than to MDA-MB-231 ( $\left.\mathrm{IC}_{50} \sim 48.5 \mu \mathrm{M}\right)$ (Table 1). On contrary, no cytotoxicity against normal breast cell MCF-10A can be detected up to $180 \mu \mathrm{M}$. Selective index (SI) was calculated based on the $\mathrm{IC}_{50}$ value of FLS on MCF10A comparing to MCF-7 or MDA-MB-231. FLS exhibited greater selectivity of MCF-7 (SI >5) cell than MDA-MB-231 $(\mathrm{SI}>3)$ against MCF-10A.

\section{Morphology observation of MCF-7 treated with FLS}

Untreated MCF-7 cells were confluent with normal morphology (Figure 2A). FLS $(36 \mu \mathrm{M})$ reduced the cell number of MCF-7 and induced cell shrinkage after 72 hours of treatment. Also, detachment of cell was also observed (Figure 2B). Scanning electron microscopy has provided a clearer comparison between ultrastructure of untreated and FLS treated MCF-7 after 72 hours of incubation. Untreated control MCF-7 was observed with normal cell morphology with extended structure and microvilli observed indicating the control cell was healthy and stably attached on the glass slide (Figure 2C). In contrast, morphological changes related to apoptosis including cell shrinkage, irregular surface, large hole, and apoptotic body were observed in the FLS treated MCF-7 (Figure 2D). In addition, apoptosis, necrosis, and viability of MCF-7 cell treated with FLS were also investigated under fluorescent microscope after staining with acridine orange and PI. Viable cell was stained as green intact cell (Figure 2E). On the other hand, MCF-7 cell treated with FLS was observed with significant increase of apoptotic population $(P<0.05)$, which was marked by chromatin condensation, cell shrinkage and membrane blebbing (Figure 2F). Based on random scoring of $\sim 200$ cells, untreated control MCF-7 cell was recorded with $>95 \%$ of viable population. Unlike control, FLS treatment induced $26 \%$ of apoptosis and $14.5 \%$ of necrosis or late apoptosis after 72 hours of incubation (Figure 2G).

\section{FLS induced $G 2 / M$ cell cycle arrest followed by apoptosis on MCF-7}

Cell cycle progression of FLS $(36 \mu \mathrm{M})$ treated MCF-7 was examined by flow cytometry PI staining assay. Result of cell cycle analysis is summarized in Figure 3. Significant $(P<0.05) \mathrm{G} 2 / \mathrm{M}$ arrest was noticed after 24 hours of FLS treatment. Subsequently, 48 and 72 hours of FLS treatment were found with drastic increase of sub $\mathrm{G}_{0} / \mathrm{G}_{1}$ population on MCF-7 indicating the possibility of DNA fragmentation to happen. To confirm the apoptotic inducing effect of FLS on MCF-7 at 48 and 72 hours, Annexin V/PI apoptosis flow cytometry was done to evaluate the late and early apoptosis indicated by externalization of phosphatidylserine with or without loss of membrane integrity (that allow PI to enter and stain the DNA), respectively. Based on the Annexin V/PI result, early apoptosis was noticed with constant increase from $9.18 \%$ to $36.72 \%$ from 24 to 72 hours. Besides, $11.86 \%$ of late apoptosis was observed after 48 hours and further increased to $17.29 \%$ at 72 hours on FLS treated MCF-7 cell (Figure 3). These results indicated that FLS treatment promoted G2/M cell cycle arrest in early time point and subsequently induced apoptosis at later time point.

\section{FLS downregulated the mRNA expression of PLKI and GLUI but upregulated the mRNA expression of WEE-I and c-Jun}

Differential expression of PLK1, WEE-1, c-Jun, and GLUT on untreated control and FLS treated MCF-7 cell was quantified by qRT-PCR assay on the cDNA synthesized from the extracted RNA. The results indicated that FLS significantly $(P<0.05)$ downregulated expression $(>2$ fold $)$ of $P L K 1$ and GLUT mRNA expression in MCF-7 cell. On the other hand, it upregulated WEE- 1 expression for all evaluated time points but only significantly increased $c$-Jun expression after 72 hours of treatment (Figure 4).

\section{FLS promoted the level of CDC2 phosphorylation, $\mathrm{Bax} / \mathrm{Bcl}-2$ ratio, $\mathrm{p} 53$, active caspases, and cytochrome $c$}

Based on the Western blot analysis, level of tumor suppressor p53 and proapoptotic Bax were upregulated in timedependent manner. Furthermore, the level of antiapoptotic protein $\mathrm{Bcl}-2$ was downregulated in the similar trend as well (Figure 5). The phosphorylation of CDC2 significantly increased with drastic reduction in $\mathrm{CDC} 2$ total protein levels at 24, 48, and 72 hours. Similarly, level of activated caspase 9 and cytochrome $\mathrm{c}$ were also detected to be significantly increased in FLS treated MCF-7 in time-dependent manner (Figure 6). 
A

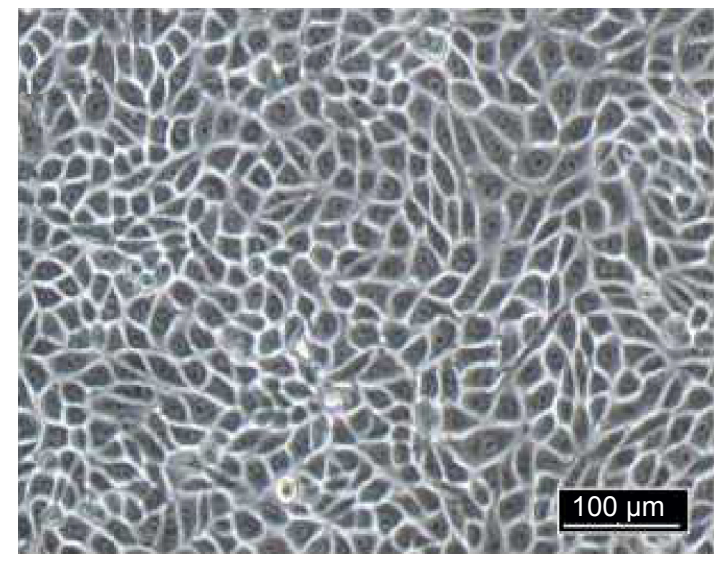

C

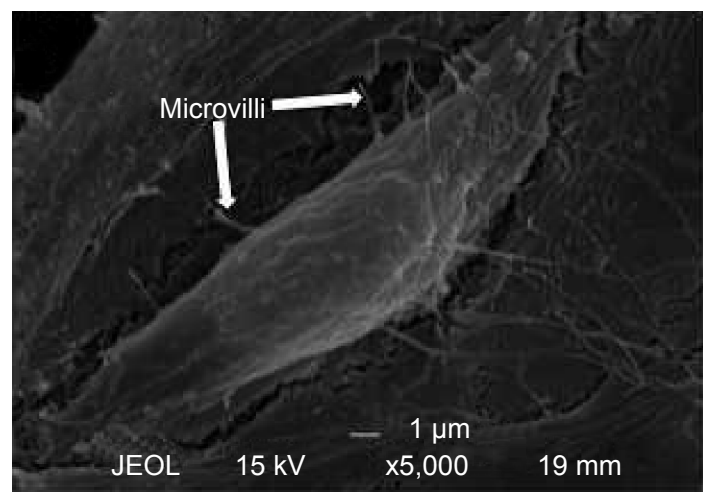

E

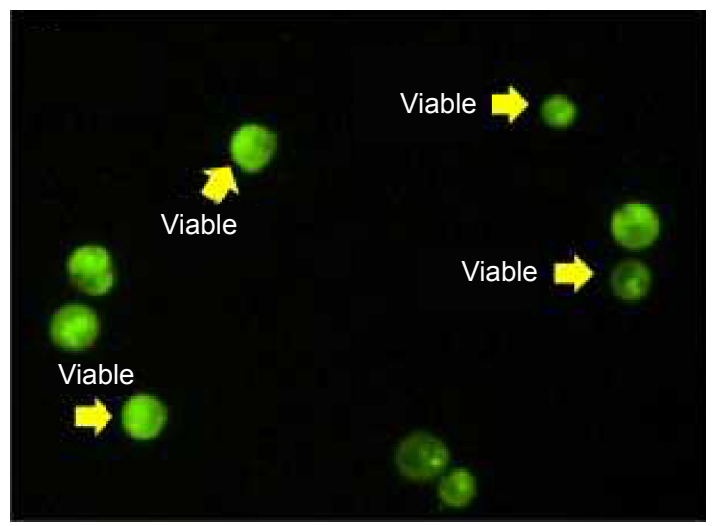

B

MCF FLS (36 $\mu \mathrm{M}), 72 \mathrm{~h}$

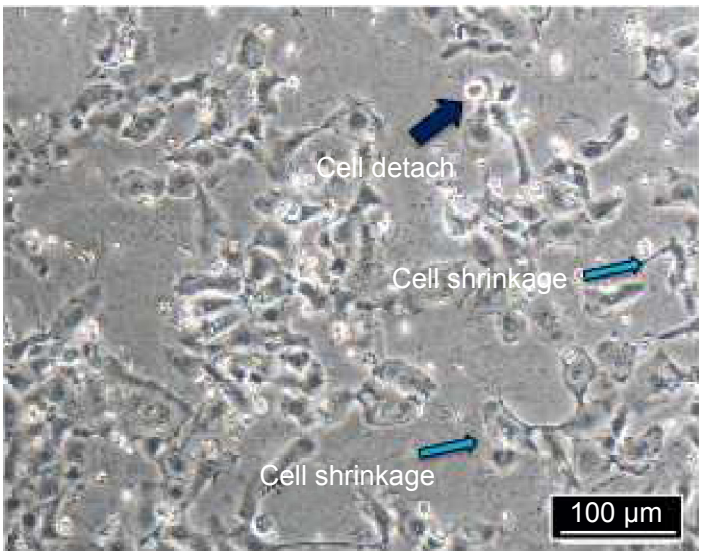

D

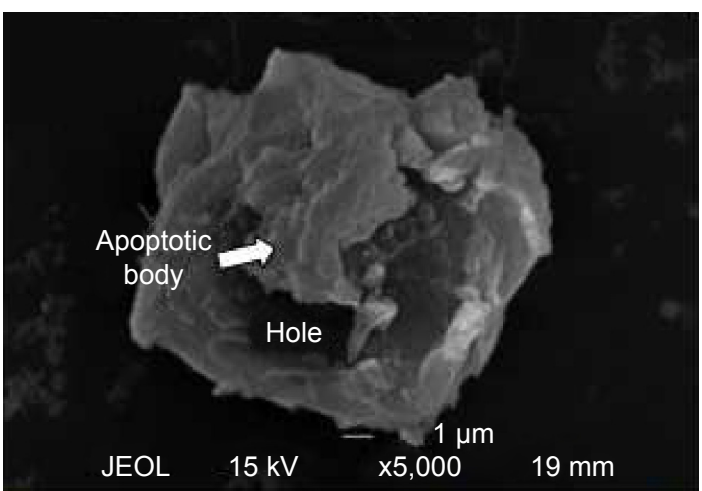

F

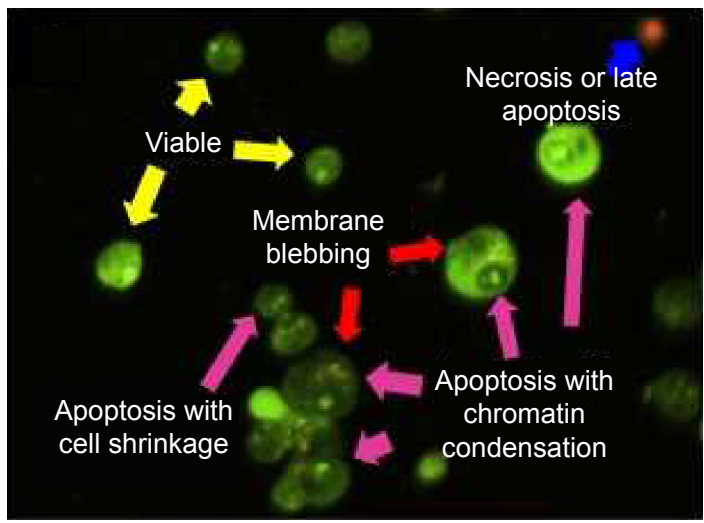

G

to Viable Apoptosis $\$$ Necrosis

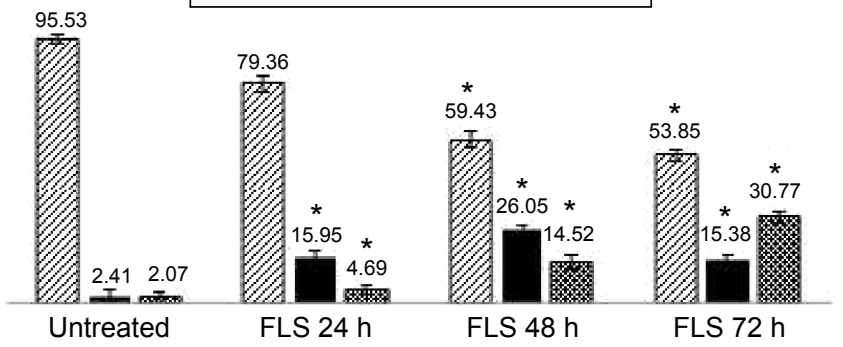

Figure 2 Light microscopic images of MCF-7.

Notes: (A) Untreated (control) and (B) FLS treated (36 $\mu$ M at 72 hours). Scanning electron microscopy images of MCF-7 (C) untreated (control) and (D) FLS treated (36 $\mu$ M at 72 hours). Acridine orange/propidium iodide (AO/PI) double staining of MCF-7 (E) untreated (control) and (F) FLS treated (36 $\mu$ M at 72 hours) and (G) Bar chart analysis of the percentage of viable, apoptotic and necrotic MCF-7 of untreated (control) and FLS treated ( $36 \mu \mathrm{M}$ at 72 hours) via AO/PI qualitative analysis at each time point. The experiments were done in triplicate and all data are expressed as mean $\pm S E$ with $(* P<0.05)$. Figures shown were representative of at least three independent replicates with similar parameters. Abbreviations: FLS: (E)-I-(2'-Hydroxy-4',6'-dimethoxyphenyl)-3-(4-methylthio)phenyl)prop-2-ene-I-one; SE, standard error of the mean. 
$I_{50}(36 \mu \mathrm{g} / \mathrm{mL})$ of FLS
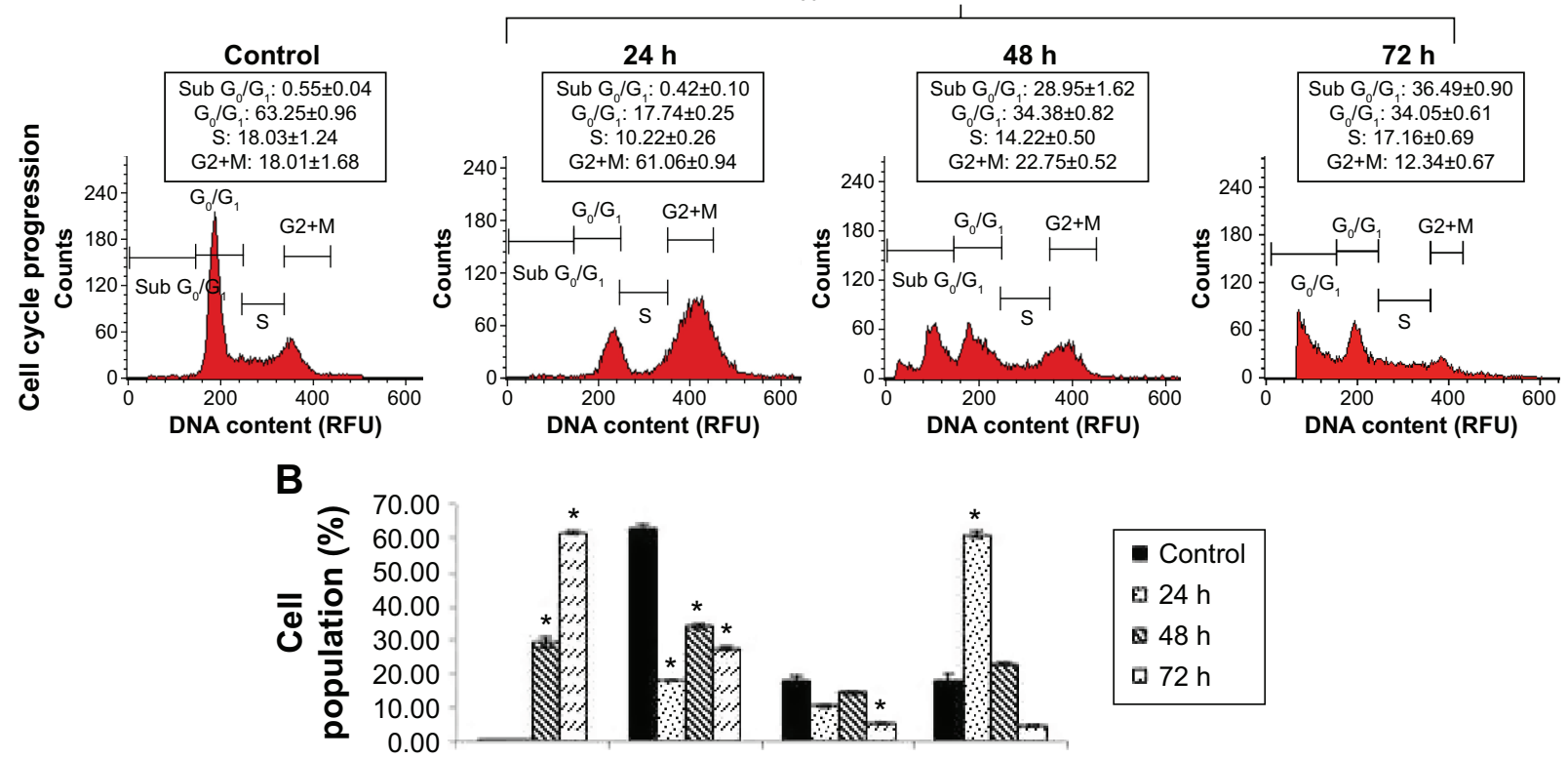

C
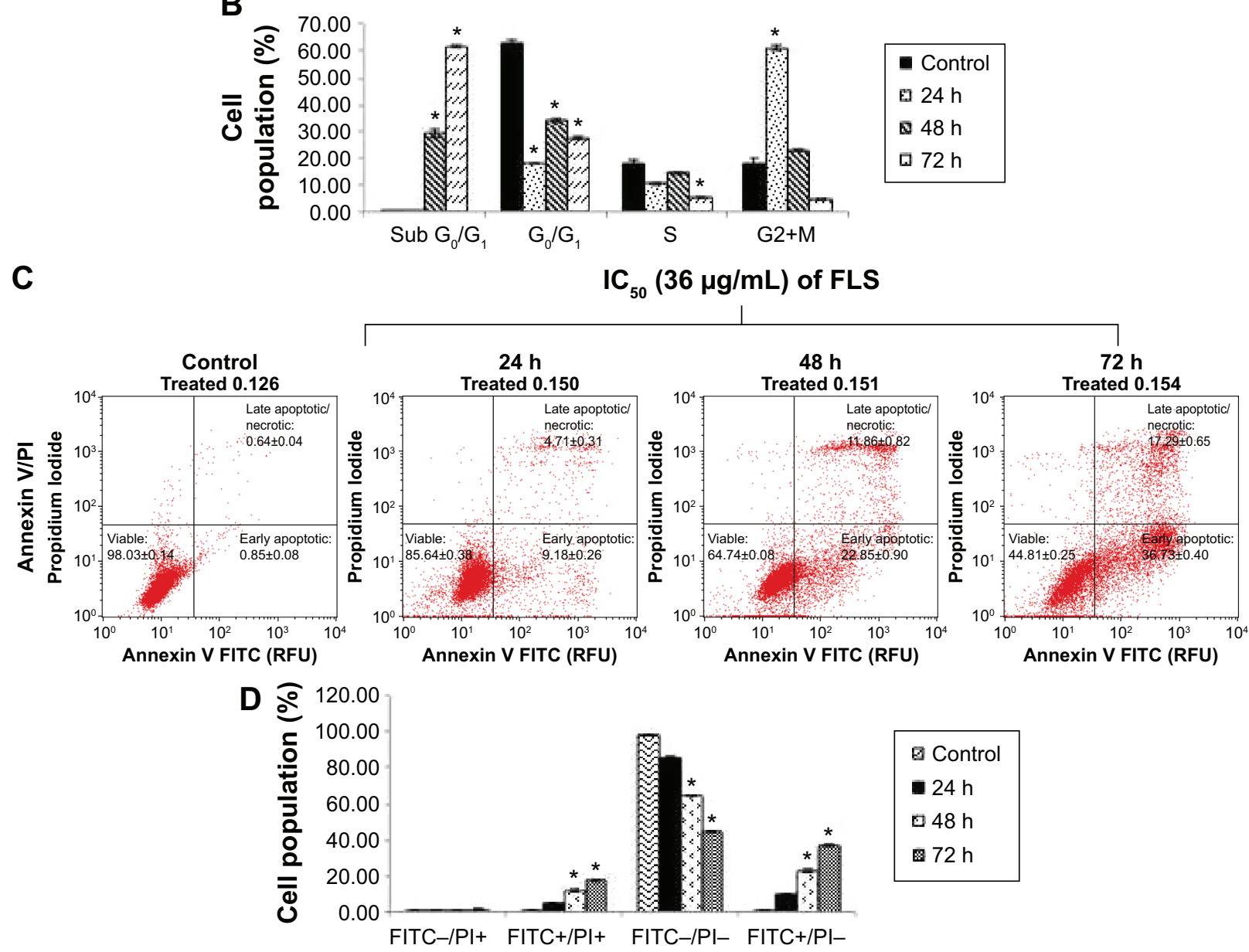

Figure 3 Flow cytometry cell cycle and Annexin V analyses of control and FLS treated MCF-7 cells.

Notes: (A) Histogram analysis of cell cycle and (B) bar chart showing the percentage of cell in each phase of the cell cycle in MCF-7 for 24,48 , and 72 hours when treated with IC $\mathrm{C}_{50}$ dose of FLS $(36 \mu \mathrm{M})$. (C) Histogram analysis of Annexin V/FITC and (D) bar chart showing the percentage of cell stained with FITC and PI in MCF-7 treated with $I C_{50}$ dose of FLS $(36 \mu \mathrm{M})$ for 24,48 , and 72 hours. The experiments were done in triplicate and all data are expressed as mean $\pm S E$ with $(* P<0.05)$. Sub-G/G, is sub-Gap0/GapI phase. IC 50 represents the concentration of FLS reduced $50 \%$ of MCF-7 cells viability.

Abbreviations: FLS: (E)-I-(2'-Hydroxy-4',6'-dimethoxyphenyl)-3-(4-methylthio)phenyl)prop-2-ene-I-one; SE, standard error of the mean; FITC+/PI+, late apoptosis population; FITC-/PI-, healthy cell population; FITC+/PI-, early apoptosis population; S, synthesis; RFU, relative fluorescence unit.

\section{Discussion}

Fighting cancer by combining knowledge of phytochemistry and synthetic chemistry has been utilized to discover potential chemotherapy agents with better selectivity against cancer than normal cell. ${ }^{7}$ Phytochemicals isolated from the natural sources have been proven with multiple bioactivities including anticancer activity but at the same time facing disadvantages such as lack of accessibility, and not being cost or time effective to obtain in large volume. These shortages can be overcome by synthetic compounds and even modified to increase the availability, bioactivities, and selectivity against cancer cell. ${ }^{14}$ 


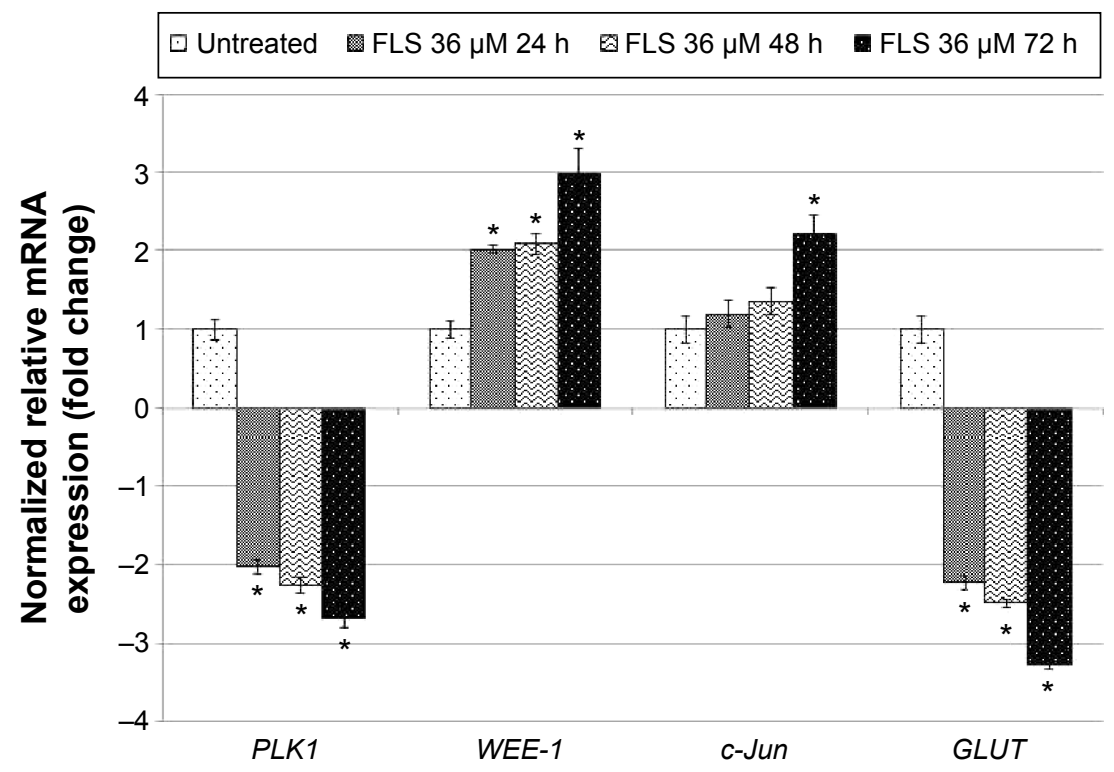

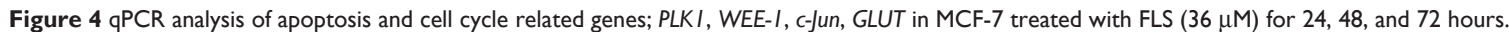

Note: The experiment was done in triplicate and the data are expressed as mean \pm SE with $(* P<0.05)$.

Abbreviations: FLS: (E)-I-(2'-Hydroxy-4', $6^{\prime}$-dimethoxyphenyl)-3-(4-methylthio)phenyl)prop-2-ene-I-one; SE, standard error of the mean; qPCR, quantitative real time polymerase chain reaction.

A
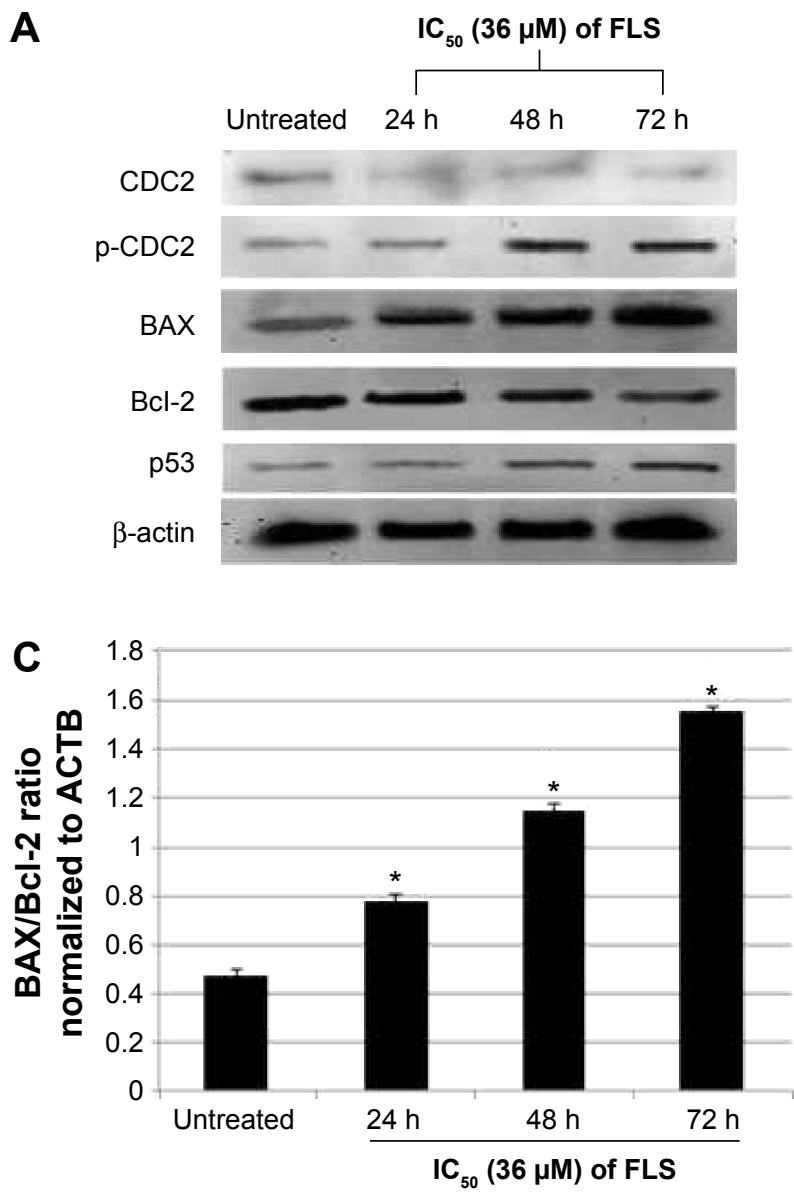

B

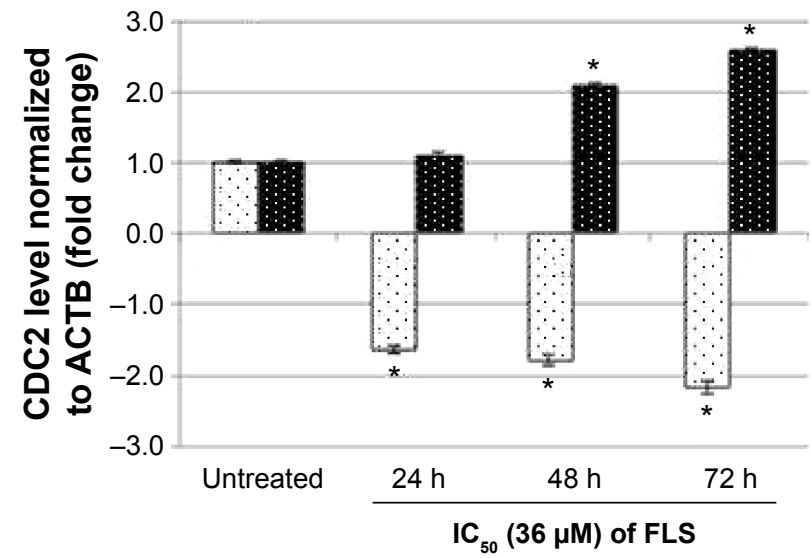

D

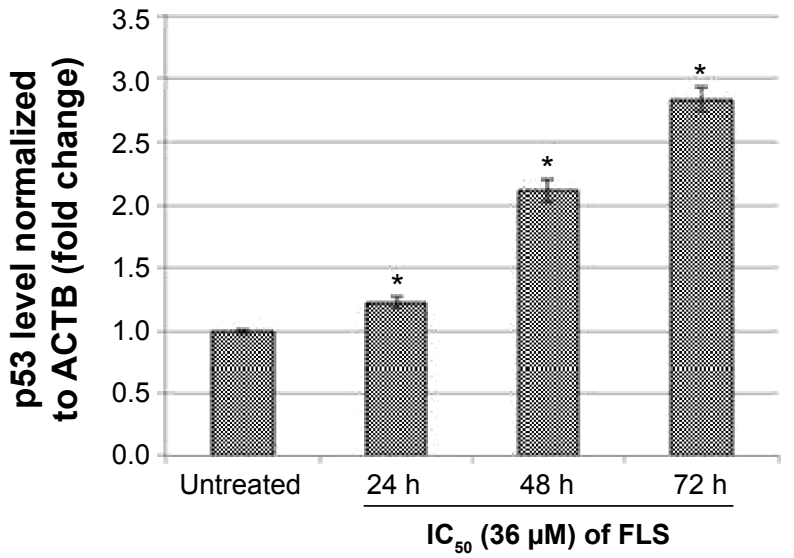

Figure 5 Differential protein expression of untreated and FLS treated MCF-7 cells.

Notes: (A) Western blot analysis of cell cycle and apoptotic-related proteins; CDC2, p-CDC2, BAX, Bcl-2, and p53 in MCF-7 treated with FLS ( $36 \mu$ M) for 24,48 , and 72 hours. Bar chart analysis of related proteins (B) CDC2 and p-CDC2, (C) ratio of BAX/Bcl-2 and (D) p53 after normalized against beta actin (ACTB). The experiment was done in triplicate and the data are expressed as mean \pm SE with $(* P<0.05)$. White bars with black dots is CDC2 while black bars with white dots is $P-C D C 2$.

Abbreviations: FLS: (E)-I-(2'-Hydroxy-4',6'-dimethoxyphenyl)-3-(4-methylthio)phenyl)prop-2-ene-I-one; SE, standard error of the mean. 


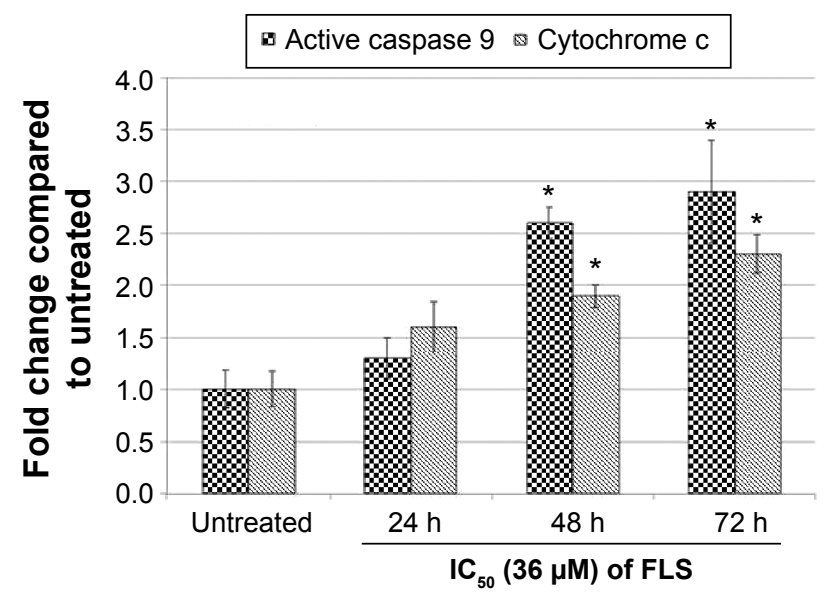

Figure 6 Detection of the activation of caspase 9 and cytochrome c in MCF-7 treated with FLS $(36 \mu \mathrm{M})$ for 24,48 , and 72 hours.

Note: The experiment was done in triplicate and the data are expressed as mean \pm SE with $(* P<0.05)$.

Abbreviations: FLS: (E)-I-(2'-Hydroxy-4',6'-dimethoxyphenyl)-3-(4-methylthio) phenyl)prop-2-ene-I-one; SE, standard error of the mean.

Several studies have shown that chalcone is an important class for the anticancer properties against various cancer cell lines. ${ }^{14}$ Previously, we have synthesized FLA and FLB and investigated their cytotoxic effects antimetastatic effects on MCF-7 and MDA-MB-231 breast cancer cell lines in vivo and in vitro. ${ }^{9,10}$ In terms of FLA ${ }^{9}$ and FLB (Abu et al), ${ }^{10}$ they possessed greater selectivity against MDA-MB-231 (SI: 5.72 for FLA and 3.66 for FLB) than MCF-7 (SI: 3.98 for FLA and 1.33 for $\mathrm{FLB}$ ) with $\mathrm{IC}_{50}$ on normal MCF-10A around 100 and $45 \mu \mathrm{M}$ for FLA and FLB, respectively. Although FLA and FLB showed good cytotoxicity against MDA-MB-231 cell, their effect on MCF-7 was just marginal with poor SI especially FLB, which has lower $\mathrm{IC}_{50}$ value on normal breast cell line. In this study, flavokawain derivative (FLS) was synthesized and subjected to cytotoxicity evaluation. MTT assay indicated that synthetic FLS was more sensitive to MCF-7 than MDA-MB-231 compared to FLA and FLB (Table 1). More interestingly, FLS did not show $\mathrm{IC}_{50}$ value $(>180)$ on normal breast MCF-10A cell thus giving the SI of 5.4 to MCF-10A/ MCF-7. However, the SI of FLS against MCF-10A/MDAMB-231 was lower than FLS against MCF-10A/MFC-7 since its cytotoxicity against MDA-MB-231 was low (Table 1). Chalcones consist of two aromatic rings ( $\mathrm{A}$ and $\mathrm{B}$ ) linked by a three-carbon unit $\alpha \beta$-unsaturated carbonyl moiety, which acts as a Michael acceptor. Apart from various substitutions on two rings, basic skeleton of chalcone (1,3-diphenyl-2-propenone) is potential in the treatment of human breast cancer. ${ }^{1,15-17}$ The difference in the structure of the compounds is the presence of methoxy $\left(\mathrm{OCH}_{3}\right)$ in FLA and $\mathrm{SCH}_{3}$ (thiomethyl group) in FLS, meanwhile no substituent is present on ring $\mathrm{B}$ of FLB. The presence of $\mathrm{SCH}_{3}$ and $\mathrm{OCH}_{3}$ on ring $\mathrm{B}$ showed the contribution against maintaining cytotoxicity on MCF-7 cell but less toxic toward normal MCF-10A cell, which possibly due to the involvement of electron donating group, makes enrich scaffold $\alpha \beta$-unsaturated carbonyl moiety to further promote apoptosis in breast cancer MCF-7 cells but without further affecting of normal breast MCF-10A cells. Previous report by Ethiraj et $\mathrm{al}^{18}$ has shown that electron donating groups in chalcones have reduced the cytotoxic activity against cancer cell. FLB, free of electron donating methoxy or thiomethyl group, was found to be more sensitive to both breast cancer MDA-MB-231 and normal MCF-10A cell lines comparing to both FLA and FLS (Table 1). On the other hand, FLS and FLA with electron donating group possessed the advantage of less toxic in breast normal MCF-10A cell thus improved the selectivity against breast cancer.

Since FLS showed better cytotoxic potential against MCF-7 cell, the mode of cell death and cell cycle arrest induced and cell cycle arrest effects were evaluated on MCF-7 using $\mathrm{IC}_{50}$ value of FLS at 48 hours $(36 \mu \mathrm{M})$. As indicated by the light microscope observation, reduction of cell number was recorded in FLS treated MCF-7 cell (Figure 2B). This result was further supported by the cell cycle analysis where G2/M arrest was observed as early as 24 hours post-FLS treatment. This effect might be contributed by the significant upregulation and downregulation of WEE-1 and $P L K-1$ in the treated cell, respectively (Figure 4). PLK1, a mitotic polo-like kinase, is a key regulator of $\mathrm{G} 2 / \mathrm{M}$ phase progression while $W E E-1$ is the nuclear kinase that inhibits the entry of cell into mitosis. ${ }^{19}$ Overexpression of PLK1 is always reported in different types of cancer including breast cancer, while downregulation of $P L K 1$ was found to link with G2/M arrest and subsequently apoptosis posttreatment with natural compound such as FLA. ${ }^{9}$ Cell cycle kinase subunit (CDC2) that binds to cyclin B1 to regulate progression from G2 to M transition was also found to be downregulated by FLS. FLS suggests that the downregulation of $\mathrm{CDC} 2$ protein level contributes to the increase of $\mathrm{CDC} 2$ phosphorylation. Suppression of $P L K 1$ and upregulation of WEE-1 were reported to contribute to the downregulation of $\mathrm{CDC} 2$ via CDC2 Tyrosine-15 phosphorylation..$^{20}$ Concomitant with this, similar trend was observed in FLS treated MCF-7 cell. Cyclin-dependent kinase inhibitor $\mathrm{p} 21$, which can be upregulated by $\mathrm{p} 53$, was promoted in FLA and FLB treated MCF-7 cell. ${ }^{9,10}$ In terms of FLS treated MCF-7 cell, although p53 was found to be upregulated (Figure 5), its role in the regulation of G2/M may be marginal because p21 was not significantly upregulated when evaluated using qRT-PCR (results not shown). Previous study has shown that G2/M 
arrest can occur with or without the involvement of tumor suppressor p53. Although the regulation of p21 in MCF-7 treated with FLS was difference comparing to FLA and FLB, all of these compounds were found to promote G2/M arrest via deregulation of $P L K 1 .{ }^{9,10,19}$

Apoptosis was significantly $(P<0.05)$ enhanced in MCF-7 after 48 and 72 hours of FLS treatment in Annexin V/PI apoptosis study. The result was further supported by the morphology observations where FLS treated MCF-7 was found with typical characteristic of apoptosis including cell detach, cell shrinkage, membrane blebbing, hole, apoptotic body, and chromatin condensation (Figure 2). FLS modulated apoptosis via upregulation of p53, Bax, caspase 9, and cytochrome c. FLS induced DNA damage associated with tumor suppressor p53 upregulation. p53 was reported as a key regulator of BCL family where its upregulation was found to promote proapoptotic BAX and suppress antiapoptotic Bcl-2, which led to secretion of cytochrome $\mathrm{c}$ that stimulates the caspase cascade activation of cell death. ${ }^{21}$ Study has shown that mitochondria played a factor in the initiation of apoptotic process by releasing cytochrome c, apoptogenic factor from the outer mitochondria membrane space into cytosol of the apoptotic cells. ${ }^{22}$ Concomitant with this, upon cytochrome c leakage, caspase 9 was being activated. This suggests that FLS induced changes in the mitochondrial membrane potential in the early stages of apoptotic cell death via mitochondrial-dependent intrinsic pathway. In this study, raised $\mathrm{BAX} / \mathrm{Bcl}-2$ ratio, cytochrome $\mathrm{c}$, and caspase 9 in the FLS treated MCF-7 indicated the involvement of FLS in promoting the intrinsic apoptosis as detected in flow cytometry and microscopic examinations. Our previous reports have shown higher sensitivity of FLA and FLB in mutant type p53 MDA-MB-231 cell than wildtype p53 MCF-7 cell. In this study, FLS was more potent in wild-type p53 MCF-7. The higher selectivity in wild-type p53 MCF-7 cell may be contributed by the exchange of $\mathrm{SCH}_{3}$ thiomethyl group in FLS. Previous study has shown that the presence of thiomethyl group improved the selectivity of the compound toward cancer cell and maintained its cytotoxicity via drastic upregulation of p53 and Bax status that subsequently promote apoptosis in leukemia cells. Similarly, regulation of FLS and this thiomethyl compound on Bcl-2 was marginal after 24 and 48 hours treatment. ${ }^{23}$

This study reveals that FLS showed good selectivity against breast cancer MCF-7 than normal MCF-10A cell line. The cytotoxicity of FLS on MCF-7 was mainly contributed by $\mathrm{G} 2 / \mathrm{M}$ cell cycle arrest and induction of apoptosis. Downregulation of $P L K 1$ and upregulation of WEE-1 associated with phosphorylation of CDC2 were the major regulators of the $\mathrm{G} 2 / \mathrm{M}$ arrest. On the other hand, mitochondrial p53 pathway was found as the contributor of the FLS induced apoptosis in MCF-7 cell. The presence of $\mathrm{SCH}_{3}$ (thiomethyl group) on ring $\mathrm{B}$ structure might play a role in cytotoxicity and apoptosis of MCF-7 cell compared to other chalcones, FLA and FLB. Thus, FLS is more potent candidate in selectively combating MCF-7 breast cancer cell. Further in vivo study shall be carried out to evaluate the antibreast cancer efficacy of FLS comparing to FLA and FLB.

\section{Acknowledgments}

This work was jointly supported by University of Malaya HIR-MoE grant (reference number - UM.C/625/1/HIR/ MOHE/CHAN/03, account number - A000003-50001) and University Malaysia Pahang internal grants no (RDU 120373 and RDU 120389).

\section{Disclosure}

The authors report no conflict of interest in this work.

\section{References}

1. American Cancer Society. Global Cancer Facts and Figures. 3rd ed. Atlanta: American Cancer Society; 2015. Available from: http://www. cancer.org/acs/groups/content/@research/documents/document/acspc044738.pdf. Accessed January 6, 2016.

2. Torre LA, Bray F, Siegel RL, Ferlay J, Lortet-Tieulent J, Jemal A. Global cancer statistics, 2012. CA Cancer J Clin. 2015;65:87-108.

3. Loganathan R, Radhakrishnan AK, Selvaduray KR, Nesaretnam K. Selective anti-cancer effects of palm phytonutrients on human breast cancer cells. RSC Advances. 2015;5:1745-1753.

4. US Mortality Data, National Center for Health Statistics, Centers for Disease Control and Prevention. American Cancer Society, Surveillance Research. Atlanta, GA: American Cancer Society; 2014.

5. Jemal A, Bray F, Center MM, Ferlay J, Ward E, Forman D. Global cancer statistics. CA Cancer J Clin. 2011;61:69-90.

6. Siegel R, Ma J, Zou Z, Jemal A. Cancer statistics, 2014. CA Cancer J Clin. 2014;64:9-29.

7. Badisa RB, Darling-Reed SF, Joseph P, Cooperwood JS, Latinwo LM, Goodman CB. Selective cytotoxic activities of two novel synthetic drugs on human breast carcinoma MCF-7 cells. Anticancer Res. 2009;29:2993-2996.

8. Batovska DI, Todorova IT. Trends in utilization of the pharmacological potential of chalcones. Curr Clin Pharmacol. 2010;5:1-29.

9. Abu N, Akhtar MN, Yeap SK, et al. Flavokawain A induces apoptosis in MCF-7 and MDA-MB231 and inhibits the metastatic process in vitro. PLoS One. 2014;9:e105244.

10. Abu N, Akhtar M, Yeap S, et al. Flavokawain B induced cytotoxicity in two breast cancer cell lines, MCF-7 and MDA-MB231 and inhibited the metastatic potential of MDA-MB231 via the regulation of several tyrosine kinases in vitro. BMC Complem Altern M. 2016;16:86.

11. Kuo YF, Su YZ, Tseng YH, Wang SY, Wang HM, Chueh PJ. Flavokawain B, a novel chalcone from Alpinia pricei Hayata with potent apoptotic activity: Involvement of ROS and GADD153 upstream of mitochondria-dependent apoptosis in HCT116 cells. Free Radic Biol Med. 2010;49:214-226.

12. Tang Y, Simoneau AR, Xie J, Shahandeh B, Zi X. Effects of the kava chalcone flavokawain A differ in bladder cancer cells with wild-type versus mutant p53. Cancer Prev Res (Phila). 2008;1:439-451. 
13. Mosmann T. Rapid colorimetric assay for cellular growth and survival: application to proliferation and cytotoxicity assays. J Immunol Methods. 1983;65:55-63.

14. Abu N, Ali NM, Ho WY, Yeap SK, Aziz MY, Alitheen N. Damnacanthal: a promising compound as a medicinal anthraquinone. Anticancer Agents Med Chem. 2014;14:750-755.

15. Go ML, Wu X, Liu X. Chalcones: an update on cytotoxic and chemoprotective properties. Curr Med Chem. 2005;12:481-499.

16. Hsu YL, Kuo PL, Tzeng WS, Lin CC. Chalcone inhibits the proliferation of human breast cancer cell by blocking cell cycle progression and inducing apoptosis. Food Chem Toxicol. 2006;44:704-713.

17. Sawle P, Moulton BE, Jarzykowska M, et al. Structure-activity relationships of methoxychalcones as inducers of heme oxygenase-1. Chem Res Toxicol. 2008;21:1484-1494.

18. Ethiraj KR, Aranjani J, Khan FN. Potential cytotoxic and apoptosis inducing agents: Synthesis and evaluation of methoxy-substituted chalcones against human lung and cervical cancers. Med Chem Res. 2013;22:5408-5417.
19. Fukasawa K. Oncogenes and tumour suppressors take on centrosomes. Nat Rev Cancer. 2007;7:911-924.

20. Fletcher L, Cheng Y, Muschel RJ. Abolishment of the Tyr-15 inhibitory phosphorylation site on cdc 2 reduces the radiation-induced $\mathrm{G}(2)$ delay, revealing a potential checkpoint in early mitosis. Cancer Res. 2002;62:241-250

21. Jiang X, Wang X. Cytochrome c-mediated apoptosis. Ann Rev Biochem. 2004;73:87-106.

22. Looi CY, Arya A, Cheah FK, et al. Induction of apoptosis in human breast cancer cells via caspase pathway by vernodalin isolated from Centratherum anthelminticum (L.) seeds. PLoS One. 2013;8:e56643.

23. Fimognari $C$, Nüsse M, Iori R, Cantelli-Forti G, Hrelia P. The new isothiocyanate 4-(Methylthio)Butylisothiocyanate selectively affects cell-cycle progression and apoptosis induction of human leukemia cells. Invest New Drugs. 2004;22:119-129.

\section{Publish your work in this journal}

Drug Design, Development and Therapy is an international, peerreviewed open-access journal that spans the spectrum of drug design and development through to clinical applications. Clinical outcomes, patient safety, and programs for the development and effective, safe, and sustained use of medicines are a feature of the journal, which has also been accepted for indexing on PubMed Central. The manuscript management system is completely online and includes a very quick and fair peer-review system, which is all easy to use. Visit http://www.dovepress.com/testimonials.php to read real quotes from published authors.

Submit your manuscript here: http://www.dovepress.com/drug-design-development-and-therapy-journal 